\title{
Philippe Askenazy, Les décennies aveugles : emploi et
} croissance, 1970-2010

Paris, Seuil, 2011

\section{Frédéric Lerais}

\section{(2) OpenEdition \\ Journals}

Édition électronique

URL : http://journals.openedition.org/travailemploi/5797

DOI : 10.4000/travailemploi.5797

ISSN : $1775-416 \mathrm{X}$

Éditeur

DARES - Ministère du Travail

\section{Édition imprimée}

Date de publication : 15 septembre 2012

Pagination : 140-142

ISSN : 0224-4365

\section{Référence électronique}

Frédéric Lerais, «Philippe Askenazy, Les décennies aveugles : emploi et croissance, 1970-2010», Travail et Emploi [En ligne], 131 | 2012, mis en ligne le 01 décembre 2012, consulté le 22 septembre 2020 URL : http://journals.openedition.org/travailemploi/5797 ; DOI : https://doi.org/10.4000/travailemploi. 5797

Ce document a été généré automatiquement le 22 septembre 2020.

(c) Direction de l'animation de la recherche, des études et des statistiques (Dares) 


\section{Philippe Askenazy, Les décennies aveugles : emploi et croissance, 1970-2010}

Paris, Seuil, 2011

Frédéric Lerais

\section{RÉFÉRENCE}

Philippe Askenazy , Les décennies aveugles : emploi et croissance, 1970-2010, Paris, Seuil, 2011, 307 p.

1 Philippe Askenazy offre un habile panorama critique de quarante ans de politiques de l'emploi. «L'ouvrage se propose de suivre pas à pas l'évolution de l'environnement et des connaissances historiques » (p. 9) : il y montre ainsi une sorte de continuum dans la construction de paradigmes pour favoriser l'emploi.

2 L'auteur traite un grand nombre de thématiques avec beaucoup de subtilité en mobilisant des approches à la fois empiriques et théoriques. C'est pour le lecteur étudiant, chercheur ou policy maker - une excellente occasion de revisiter quelques bégaiements de l'histoire des politiques de l'emploi. Les matériaux mobilisés sont variés: Askenazy ne se contente pas d'analyser des séries statistiques mais convoque également des informations d'époque en utilisant notes administratives internes et littérature économique (par la relecture d'anciens numéros d'Économie et statistique de l'Insee et des Perspectives de l'emploi de l'OCDE). Cette diversité de sources permet de porter un regard différent de celui que l'on a à la seule lecture des séries statistiques car on peut ainsi réviser l'analyse qui était faite à l'époque. Ne serait-ce que pour cette riche mobilisation, il faut conseiller la lecture de l'ouvrage dans son entier et non de ses seuls résumés. 


\section{Des dispositifs qui ont (dé)structuré le fonctionnement de l'économie}

3 L'auteur signale les tournants et les continuités sur quatre décennies. Notons, du côté des tournants, celui des années 1986-1993 avec la percée libérale. Askenazy montre que le changement de théories économiques sur la période, avec l'arrivée des modèles d'appariement et d'hystérèse, participe au développement idéologique des politiques : c'est le temps de l'ordonnance de 1986 qui libéralise l'usage des contrats à durée déterminée (CDD) et du temps partiel, de la fin de l'autorisation administrative de licenciement et des prémisses de la mise en place d'exonérations de cotisations sociales employeurs pour créer des emplois. C'est aussi le temps de la privatisation et de la déréglementation bancaire. La France se singularise, par le fait qu'il n'y a pas eu, dans le même temps, d'objectif de développement de l'industrie. À la même époque, le Royaume-Uni, en pleine percée libérale, avait une politique de développement de son industrie financière. On assiste alors à « une perte de compétitivité » : Askenazy revient sur cette thèse par petites touches à de nombreuses reprises.

4 L'auteur souligne aussi très bien les effets structurants sur l'économie des préconisations de politique d'emploi. La période 1986-93 est également marquée par la baisse de la durée du travail sous l'effet du développement des politiques incitatives au temps partiel. L'idée sous-jacente est qu'il y a un gisement d'emplois à temps partiel à exploiter. Les politiques appliquées ont à terme des effets profonds dans le secteur du commerce en adaptant les calendriers de travail à la fréquentation des magasins en même temps qu'en autorisant la forte parcellisation du temps de travail des individus. La mesure d'incitation au développement du temps partiel a certes disparu mais le mode de production est resté identique pour les magasins. Des dispositifs ont ainsi été empilés qui ont continué de «façonner » le fonctionnement économique du marché du travail.

5 L'auteur remet souvent en cause le rôle des experts. Ainsi, Askenazy rappelle qu'à partir de 1993, les experts plaident, de manière quasi-consensuelle, pour une nouvelle politique de l'emploi qui reflète globalement la stratégie pour l'emploi de l'OCDE. Ce consensus repose sur un diagnostic commun: sous l'effet de la mondialisation et des technologies, les compétences s'accroissent et renforcent les inégalités de salaire, sans pour autant provoquer d'effets positifs sur l'emploi (p. 147). En France, la politique se concentre sur la baisse du coût du travail et prend la forme d'un allégement de cotisations sociales général: en 1993, le gouvernement Juppé lance une politique d'allégement de cotisations sociales sur les bas salaires (puisqu'il n'est pas possible de mener une politique de baisse du coût du travail des non-qualifiés).

\section{Errements des politiques et préconisations}

6 Finalement, l'auteur souligne, à juste titre, que tout cela ne s'est pas traduit par une forte politique d'innovation et de politique industrielle : à l'inverse des États-Unis où les politiques d'innovation ont fait l'objet de théorisation(s), la France a, en quelque sorte, raté le train de l'économie de la connaissance : « obnubilée par le développement de l'emploi peu qualifié, que les experts appelaient de leurs vœux, et aveuglée par une croissance retrouvée et un chômage contenu, la France n'entre pas pleinement dans la société de la connaissance » (p. 186). 
Dans sa conclusion, Askenazy signale que la politique de l'emploi a peut-être conduit à sur-réagir et que les experts ont été entraînés à abandonner certaines questions pourtant cruciales comme les salaires pour tenter de faire face à la dégradation du marché du travail (p. 277). Il y a, selon lui, urgence à bâtir une autre politique, qui empile moins de dispositifs, à réfléchir en termes de besoins et plaide pour une politique qui prenne le contre-pied des politiques «barristes » qui auraient finalement abandonné les moins qualifiés à leur sort : il défend ainsi des politiques d'éducation qui démarrent dès la petite enfance afin d'éviter les pièges de la reproduction des inégalités. Il met aussi l'accent sur les questions des secteurs de santé, qui doivent être appréhendées non pas comme un coût, mais comme un facteur de bien-être. Les principaux débats concernant la santé portent sur les « dérives de dépenses » de santé et leur impact sur les comptes de la sécurité sociale. La question de l'atout que représente le développement du secteur de la santé en termes de bien-être individuel, d'allongement de l'espérance de vie et donc de progrès social est pour le moins occultée.

8 En somme, cet ouvrage critique mérite une lecture attentive parce qu'il met en perspective les politiques économiques du moment. Le constat qui en ressort est sévère : il y a finalement assez peu d'innovation en matière de politiques de l'emploi. Le rapport Mattéoli (1993) sur «les obstacles structurels à l'emploi» est à ce titre exemplaire dans la mesure où on y retrouve les « innovations » les plus récentes telles que la piste de TVA sociale (mais la contribution sociale généralisée [CSG] sera préférée), la préconisation de suppression des CDD remplacés par un contrat unifié, etc.

Il reste deux bémols à formuler. D'abord, l'exercice de panorama de quarante ans de politiques de l'emploi en trois cents pages fait que l'auteur doit parfois passer trop vite sur les discussions des résultats des études mobilisées. C'est notamment le cas sur les travaux scientifiques d'évaluation des mesures "coûteuses" d'allégement de cotisations sociales et des 35 heures (p. 173). Il en est de même de la comparaison des politiques envers les jeunes en France et au Royaume-Uni. Enfin, au fil du texte, l'auteur signale des réseaux, et égraine des noms, mais la généalogie des idées y est imparfaite. "Les principaux organes de conseil économique du gouvernement sont composés des mêmes experts issus des mêmes institutions qui influencent la politique française depuis quarante ans » (p. 248) : voilà qui aurait mérité une enquête un peu plus approfondie.

\section{AUTEURS}

\section{FRÉDÉRIC LERAIS}

Institut de recherches économiques et sociales (IRES) 